\title{
Image Morphing: A Literature Study
}

\author{
Harmandeep Singh \\ Sliet University \\ Sangrur, India
}

\author{
Amandeep Kumar \\ Sliet University \\ Sangrur, India
}

\author{
Gurpreet Singh \\ Punjabi University \\ Patiala, India
}

\begin{abstract}
Image morphing has been the subject of much attention in recent years. It has proven to be a powerful visual effects tool in film and television, depicting the fluid transformation of one digital image into another. This paper reviews the growth of this field and describes recent advances in image morphing in terms of three areas: feature specification, warp generation methods, and transition control. These areas relate to the ease of use and quality of results. We will describe the role of radial basis functions, thin plate splines, energy minimization, and multilevel free-form deformations in advancing the state-of-the-art in image morphing. A comparison of various techniques for morphing one digital image in to another is made. We will compare various morphing techniques such as Feature based image morphing, Mesh and Thin Plate Splines based image morphing based on different attributes such as Computational Time, Visual Quality of Morphs obtained and Complexity involved in Selection of features. We will demonstrate the pros and cons of various techniques so as to allow the user to make an informed decision to suit his particular needs. Recent work on a generalized framework for morphing among multiple images will be described.
\end{abstract}

Keywords:Morphing, feature extraction, warping, transition control, mesh-warping, thin plate splines.

\section{INTRODUCTION}

Morphing can be defined as an animated transformation of one image into another image. Morphing involves image processing techniques like warping and cross dissolving. Cross dissolving means that one image fades to another image using linear interpolation. This technique is visually poor because the features of both images are not aligned, and that will result in double exposure in misaligned regions. In order to overcome this problem, warping is used to align the two images before cross dissolving. Warping determines the way pixels from one image are correlated with corresponding pixels from the other image. It is needed to map the important pixels, else warping doesn't work. Moving other pixels is obtained by extrapolating the information specified for the control pixels. Knowing cross dissolving is very simple; the real problem of morphing becomes the warping technique. Morphing is actually a cross dissolving applied to warped images. Warping techniques vary in the way the mapping of control pixels is specified and the interpolating technique that is used for other pixels[5.6,7].

Morphing applications are very easy to find. Film makers from Hollywood use advanced morphing techniques to generate special effects. Even Disney animations are made using morphing, for speeding production. Because there are a small number of applications to generate face morphing, there is an increased interest in this domain.

\section{MORPHING PRINCIPLE}

Image morphing combines image warping with a method that controls the color transition in the intermediate images produced. To morph one image to another, new positions and color transition rates for the pixels in each of the images in the sequence must be calculated. Three processes are involved in this method
(i)
(ii)
Feature specification
(iii)
Warp generation
Transition control

\subsection{Feature Specification:}

Feature specification is the most tedious aspect of morphing. Although the choice of allowable primitives may vary, all morphing approaches require careful attention to the precise placement of primitives. Given feature correspondence constraints between both images, a warp function over the whole image plane must be derived. This process, which we refer to as warp generation, is essentially an interpolation problem. Another interesting problem in image morphing is transition control. If transition rates are allowed to vary locally across in between images, more interesting animations are possible.

\subsection{Warp Generation:}

Warp generation is an algorithm that calculates and transforms the pixels in one image to new positions in the other image. Many algorithms have already been proposed to do warping. Once the pixels are in position, transition control blends in the colors between the two images. Transition control has also received a lot of attention. Originally, crossdissolve was the color blending method of choice, but this method produced undesirable artifacts referred to as "ghosts". 


\subsection{Transition Control:}

Transition control determines the rate of warping and color blending across the morph sequence. If transition rates differ from part to part in in between images, more interesting animations are possible. Such non uniform transition functions can dramatically improve the visual content.

\section{IMAGE MORPHING TECHNIQUES}

\subsection{Cross Dissolve Morphing}

Before the development of morphing, image transitions were generally achieved through the use of cross-dissolves, e.g., linear interpolation to fade from one image to another .Figure. 1 depicts this process applied over five frames. The result is poor, owing to the double-exposure effect apparent in misaligned regions. This problem is particularly apparent in the middle frame, where both input images contribute equally to the output. Morphing achieves a fluid transformation by incorporating warping to maintain geometric alignment throughout the cross-dissolve process.

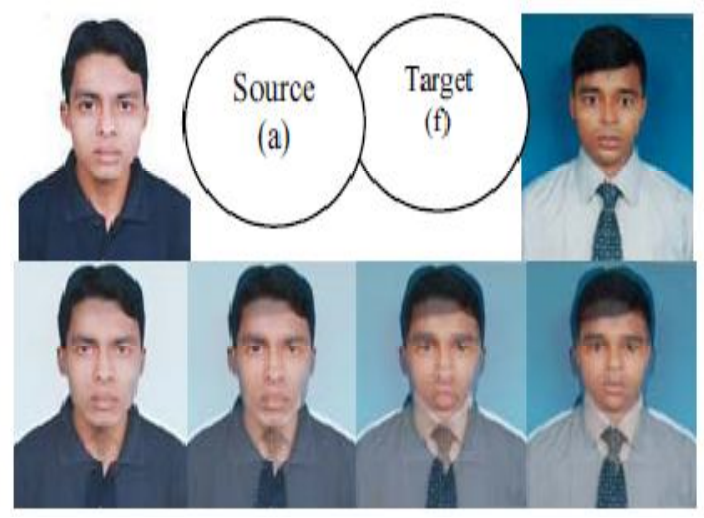

Figure.-1 (a-f).Example of cross-dissolve morphing [4].

\subsection{Mesh Warping}

Mesh warping was pioneered at Industrial Light \& Magic (ILM) by Douglas Smythe for use in the movie Willow in 1988. It has been successfully used in many subsequent motion pictures[1]. To illustrate the 2-pass mesh warping algorithm, consider the image sequence shown in Fig.-2. The five frames in the middle row represent a metamorphosis (or morph) between the two faces at both ends of the row[1]. We will refer to these two images as IS and IT, the source and the target images, respectively. 'fivesource image has mesh MS associated with it that specifies the coordinates of control points, or landmarks. A second mesh, MT, specifies their corresponding positions in the target image. MeshesMS and MT are respectively shown overlaid on Isand IT in the upper left and lower right images of the figure. Noticethat landmarks such as the eyes, nose, and lips lie belowcorresponding grid lines in both meshes. Together, MS and MT are used to define the spatial transformation that mapsall points in IS onto IT. The meshes are constrained to betopologically equivalent, i.e., no folding or discontinuitiesare permitted. Therefore, the nodes in MT may wander as far from MS as necessary, as long as they do not causeself-intersection. Furthermore, for simplicity, the meshes are constrained to have frozen borders. All intermediate frames in the morph sequence are the product of a 4-step process:

For each frame fdo linearly interpolate mesh M, between MS and MT warp ISto I I, using meshes MS and M warpIT to 12 , using meshes MT and M linearly interpolate image I $\mathrm{f}$, between I1 and I2 end

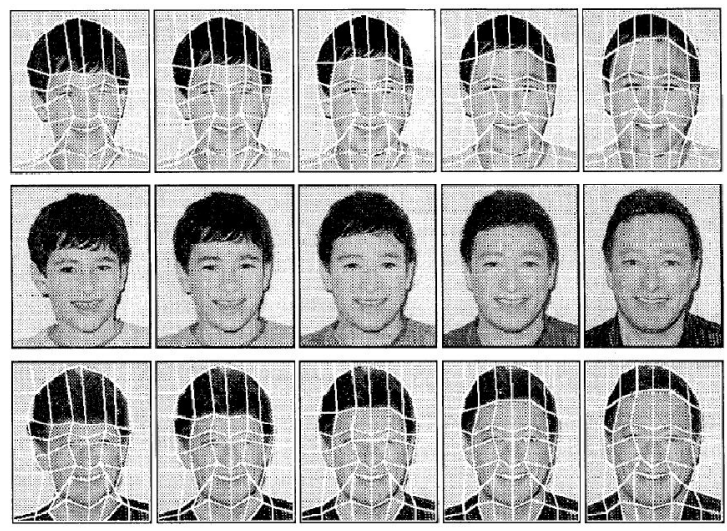

Figure.-2: Mesh warping[1]

Figure.-2 depicts this process. In the top row of the figure, mesh MS is shown deforming to mesh MT, producing an intermediate meshM for each frame JF. Those meshes are used to warp IS into increasingly deformed images, thereby deforming IS from its original state to those defined by the intermediate meshes. The identical process is shown in reverse order in the: bottom row of the, figure, where IT is shown deforming from its original state. The purpose of this procedure is to maintain the alignment of landmarks between IS and IT as they both deform to some intermediate state, producing the pair of I1 and I2 images shown in the top and bottom rows, respectively. Only after this alignment is maintained does a cross-dissolve between successive pairs of I1 and 12 become meaningful, as shown in the morph sequence in the middle row. This sequeince was produced by applying the weights $[1, .75, .5, .25,0]$ and $[0, .25, .5, .75,1]$ to the five images in the top and bottom rows, respectively, and adding the two sets together. This process demonstrates that morphing is simply a cross-dissolve applied to warped imagery. The important role that warping plays here is readily apparent by (comparing the morph sequence in Figure.-2 with the cross-dissolve result in Figure.-1. The use of meshes for feature specification facilitates a straightforward solution for warp generation: bicubic spline interpolation.

\subsection{Field Morphing}

While meshes appear to be a convenient manner of specifying pairs of feature points, they are, however, sometimes cumbersome to use[1]. The field morphing algorithm developed by Beier and Neelyat Pacific Data Images grew out of the desire to simplify the user interface to handle correspondence by means of line pairs. A pair of 
corresponding lines in the source and target images defines a coordinate mapping between the two images. In addition to the straightforward correspondence provided for all points along the lines, the mapping of points in the vicinity of the line can be determined by their distance from the line. Since multiple line pairs are usually given, the displacement of a point in the source image is actually a weighted sum of the mappings due to each line pair, with the weights attributed to distance and line length. This approach has the benefit of being more expressive than mesh warping. For example, rather than requiring the correspondence points of Fig. 3 to all lie on a mesh, line pairs can be drawn along the mouth, nose, eyes, and cheeks of the source and target images. Therefore only key feature points need be given. Although this approach simplifies the specification of feature correspondence, it complicates warp generation. This is due to the fact that all line pairs must be considered before the mapping of each source point is known. This global algorithm is slower than mesh warping, which uses bicubic interpolation to determine the mapping of all points not lying on the mesh. A more serious difficulty, though, is that unexpected displacements may be generated after the influence of all line pairs are considered at a single point. Additional line pairs must sometimes be supplied to counter the ill-effects of a previous set. In the hands of talented animators, though, the mesh warping and field morphing algorithms have both been used to produce startling visual effects.

\subsection{Radial Basis Functions / Thin Plate Splines}

Thin-plate Spline is a conventional tool for surface interpolation over scattered data. It is an interpolation method that finds a "minimally bended" smooth surface that passes through all given points. The name "Thin Plate" comes from the fact that a TPS more or less simulates how a thin metal plate would behave if it was forced through the same control points. Let us denote the target function values $v i$ at locations $(x i, y i)$ in the plane, with $\mathrm{i}=1,2, \ldots \ldots . \mathrm{p}$, where $\mathrm{p}$ is the number of feature points. In particular, we will set $v i$ equal to the coordinates ( $\left.x i^{\prime}, y i^{\prime}\right)$ in turn to obtain one continuous transformation for each coordinate. An assumption is made that the locations $(x i, y i)$ are all different and are not collinear[3].

The Figure-3.is a simple example of coordinate transformation using TPS. It starts from two sets of points for which it is assumed that the correspondences are known (a). The TPS warping allows an alignment of the points and the bending of the grid shows the deformation needed to bring the two sets on top of each other (b). In the case of TPS applied to coordinate transformation we actually use two splines, one for the displacement in the $\mathrm{x}$ direction and one for the displacement in the $y$ direction. The two resulting transformations are combined into a single mapping. (a)

(b)

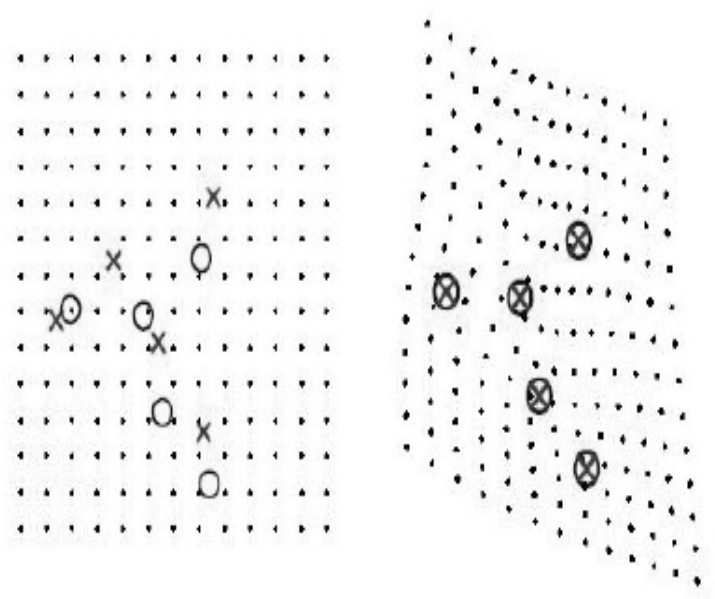

Figure-3: Example of coordinate transformation using TPS[4].

\subsection{Energy Minimization}

All of the methods described above do not guarantee the oneto-one property of the generated warp functions. When a warp is applied to an image, the one-to-one property prevents the warped image from folding back upon itself. An energy minimization method has been proposed for deriving one-toone warp functions in. That method allowsm extensive feature specification primitives such as points, polylines, and curves. Internally, all primitives are sampled and reduced to a collection of points. These points are then used to generate a warp, interpreted as a $2 \mathrm{D}$ deformation of a rectangular plate. A deformation technique is provided to derive $\mathrm{C}^{\prime}$-continuous and one-to-one warps from the positional constraints. The requirements for a warp are represented by energy terms and satisfied by minimizing their sum[1]. The technique generates natural warps since it is based on physically meaningful energy terms. The performance of this method, however, is hampered by its high computational cost.

\subsection{Multi-Level Free-Form Deformation}

A new warp generation method was presented in this chapter that is much simpler and faster than the related energy minimization method[1]. Large performance gains are achieved by applying multilevel free-form deformation (MFFD) across a hierarchy of control lattices to generate oneto-one and $\mathrm{C} 2$-continuous warp function. In particular, warps were derived from positional constraints by introducing the MFFD as an extension to free-form-deformation. In that paper, the bivariate cubic B-spline tensor product was used to define the FFD function. A new direct manipulation technique for FFD, based on 2D B-spline approximation, was applied to a hierarchy of control lattices to exactly satisfy the positional constraints. To guarantee the one-to-one property of a warp, a sufficient condition for a cubic B-spline surface to be one-toone was presented. The MFFD generates $\mathrm{C} 2$-continuous and one-to-one warps which yield fluid image distortions. The 
MFFD algorithm was combined with the energy minimization method of in a hybrid approach. An example of MFFD-based morphing is given in Figure.-4.

Notice that the morph sequence shown in the middle row of the figure is virtually identical to that produced using mesh warping in Figure.-2. The benefit of this approach, however, is that feature specification is more expressive and less cumbersome. Rather then editing a mesh, a small set of feature primitives are specified. To further assist the user, snakes are introduced to reduce the burden of feature specification. Snakes are energy minimizing splines that move under the influence of image and constraint forces. They were first adopted in computer vision as an active contour model. Snakes streamline feature specification because primitives must only be positioned near the features. Image forces push snakes toward salient edges, thereby refining their final positions and making It possible to capture the exact position of a feature easily and precisely.
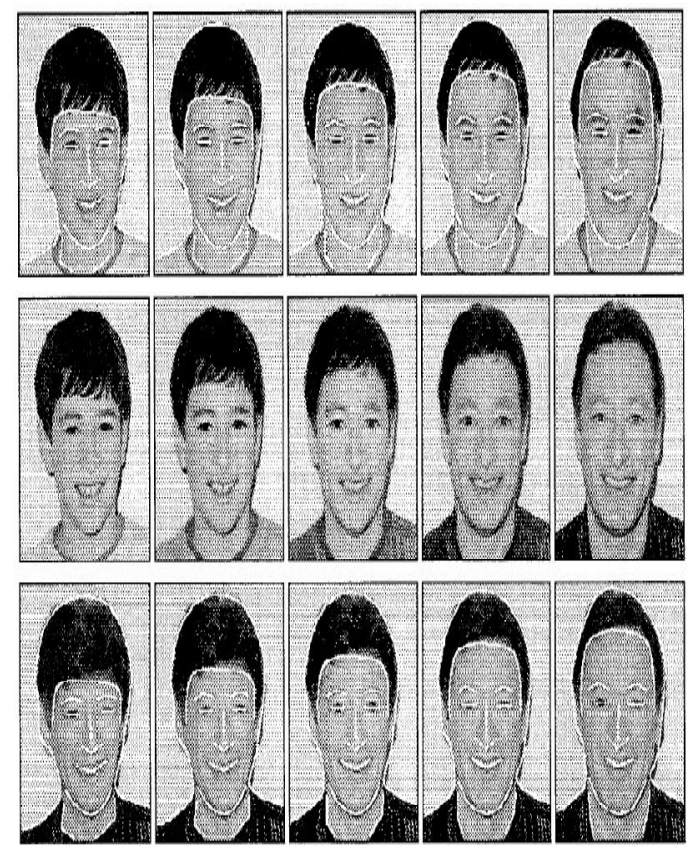

Figure-4. MFFD-based morphing[1]

\section{COMPARISON}

When we compared the three algorithms on a scale of computational speed, we found the Mesh Warping algorithm to be the best $[2,8]$. This results from the fact that the region is divided into a mesh and each mesh patch essentially has a local region of influence. Hence the computation is localized and independent, thus allowing for high level of parallelism. But a word of caution is in place. The computational advantage of the Mesh Warping algorithm is greatly offset by the huge amount of time overhead required to select mesh nodes all over the image. The main disadvantage of the Feature-based and Thin Plate Spline algorithms is speed. As the warping here is global the entire set of feature lines/control pixels that are specified need to be referenced for each pixel. As a result amount of time taken for each frame is proportional to the product of the number of pixels in the image and the number of control lines/pixels used. Table-1 gives the average warping time for each of our algorithms.

Table-1: Table showing the Comparison of different warping techniques[2]

\begin{tabular}{|l|l|}
\hline $\begin{array}{l}\text { Algorithm } \\
\text { Name }\end{array}$ & Computation Time \\
\hline $\begin{array}{l}\text { Mesh } \\
\text { Warping }\end{array}$ & $\begin{array}{l}0.15 \mathrm{~s} \text { with a 10X10 } \\
\text { mesh }\end{array}$ \\
\hline $\begin{array}{l}\text { Feature- } \\
\text { based } \\
\text { Warping }\end{array}$ & $\begin{array}{l}0.75 \mathrm{~s} \text { with } 11 \text { feature } \\
\text { lines }\end{array}$ \\
\hline $\begin{array}{l}\text { Thin Plate } \\
\text { Spline } \\
\text { Warping }\end{array}$ & $\begin{array}{l}0.45 \mathrm{~s} \text { with } 5 \text { control } \\
\text { points }\end{array}$ \\
\hline
\end{tabular}

Finally we wish to put in a word on the individual advantages and disadvantages of the three algorithms. The mesh warping algorithm requires that all four edges of the source and destination image be frozen. But this seemingly limiting constraint provides the mesh warping algorithm with its simplicity of implementation. The mesh warping algorithm also requires that the source and destination meshes be topologically equivalent i.e. no folding or discontinuities. This all adds to the problem of selecting mesh nodes spread through out the image. In case of the feature-based warping algorithm, sometimes unexpected and unwanted interpolations are generated due to some line combinations. Additional image processing efforts are required to fix these distortions and improve the quality of results.

\section{FUTURE WORK}

The traditional formulation for image morphing considers only two input images at a time, i.e., the source and target images. In that case, morphing among multiple images is understood to mean a series of transformations from one image to another. This limits any morphed image to take on the features and colors blended from just two input images.

Given the success of morphing using this paradigm, it is reasonable to consider the benefits possible from a blend of more than two images at a time. For instance, consider the generation of a facial image that is to have its eyes, ears, nose, and profile derived from four different input images. In this case, morphing among multiple images is understood to mean a seamless blend of several images at once. Despite the explosive growth of morphing in recent years, the subject of morphing among multiple images has been neglected.

In ongoing work conducted by the author and his colleagues, a general framework is being developed that extends the traditional image morphing paradigm applied to two images. 
We formulate each input image to be a vertex of a regular convex polyhedron in $(\mathrm{n}-1)$-dimensional space, where $\mathrm{n}$ is the number of input images. An in between (morphed) image is considered to be a point in the convex polyhedron. The barycentric coordinates of that point determine the weights used to blend the input images into the In between image. Morphing among multiple images is ideally suited for image composition applications where elements are seamlessly blended from two or more images. A composite image is treated as a metamorphosis of selected regions in several input images. The regions seamlessly blend together with respect to geometry and color.

In future work, we will determine the extent to which the technique produces high quality composites with considerably less effort than conventional image composition techniques. In this regard, the technique can bring to image composition what image warping has brought to cross-dissolve in deriving morphing: a richer and more sophisticated class of visual effects that are achieved with intuitive and minimal user interaction.

Future work in morphing will also address the automation of morphing among limited classes of images and video sequences. Consider a limited, but common, class of images such as facial images. It should be possible to use computer vision techniques to automatically register features between two images. Model-based vision should be able to exploit knowledge about the relative position of these features and automatically locate them for feature specification. Currently, is is an active area of research, particularly for compression schemes designed for videoconference applications. The same automation applies to morphing among two video sequences, where time varying features must be tracked[9,10,11].

\section{CONCLUSION}

The focus of this article has been to survey various morphing algorithms and provide the animator with sufficient information to make an informed choice suiting his particular needs. In doing so we have defined a few easily comparable attributes, such as visual quality of morph, the ease with which the animator can select control pixels and the computational complexity. We found that Mesh morphing gives the best result among the algorithms we implemented but it requires a significant amount of animator effort in selecting the control pixels. The Thin Plate Spline gives results, which are of comparable quality with very little effort required from the animator. The Feature based morphing algorithm requires the animator to select a significantly larger number of feature lines to give the same results.
In summary, we feel that the Thin Plate Spline warping based Image Morphing algorithm is the best choice since it produces good quality results for lest animator effort. There are a variety of Image Morphing algorithms such as Image Morphing with snakes and free formed deformations, Image morphing using deformable surfaces, Image morphing using Delaunay triangulation and many others besides. Due to paucity of resources and time, we are unable to provide a comprehensive comparison of these algorithms.

\section{REFERENCES}

[1] George Wolberg, "Recent Advances in Image Morphing", Department of Computer Science City College of New York / CUNY New York, NY 10031

[2] Prashant K. Oswal and Prashanth Y. Govindaraju, "Image Morphing: A Comparative Study", Department of Electrical and Computer Engineering, Clemson University, Clemson

[3 AlexandruVlad FECIORESCU, "Image Morphing Techniques", JUNE 2010 1 12 NUMBER 5 JIDEG

[4] Md. TajmilurRahman*, Al-Amin, M. A. Jobayer Bin Bakkre, Ahsan Raja Chowdhury $\dagger$ and Md. Al-Amin Bhuiyan¥, "A Novel Approach of Image Morphing Based on Pixel Transformation". 1-4244-15519/07/\$25.00 (C2007 IEEE.

[5] Mu-Chun Su and I-Chen Liu, "Facial Image Morphing by Self-organizing Feature Maps", Department of Electrical Engineering, Tamkang University, Taiwan, R.O.C., 07803-5529-6/99/\$10.00 01999 IEEE.

[6] StephenMullens and Simon Notley, "An Introduction to image morphing", 2006.

[7] T. Beier and S. Neely, "Feature-bssed image metmoxpiiosis", Compter Graphics (Proc. SIGGHAPH '92), 26(2):35-42, 1992.

[8] S.-Y Lee, K.-E: Chwa, S. Y Shin, and G. Wolberg. Image metamorphosis using snakes and free-form deformations. Computer Graphics (Proc.SIGGRAPH'95), pages 439448, 1995.

[9] Mu-Chun Su and I-Chen Liu, "Facial Image Morphing by Self-organizing Feature Maps", Department of Electrical Engineering, Tamkang University, Taiwan, R.O.C. 07803-5529-6/99/\$10.00 01999 IEEE.

[10] Henry Johant Yuichi KoisotTomoyukiNishitat, "Morphing Using Curves and Shape Interpolation Techniques", Dept. of Information Science, Dept. of Complexity Science and Engineering University of Tokyo, 0-7695-0868-5/0\$01 0.0002000 IEEE.

[11] Stephen Karungaru, Minoru Fukumi and Norio Akamatsu, "MORPHING FACE IMAGES USING AUTOMATICALLY SPECIFIED FEATURES", University of Tokushima, 2-1 Minami Josamjima 7708506, Tokushima, Japan., 0-7803-8294-3/04/\$20.00 @)2004 IEEE 741. 